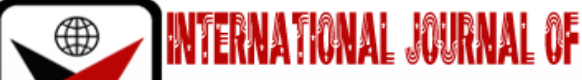

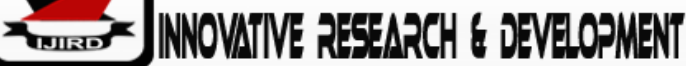

ISSN 2278-0211 (Online)

\section{Experimental Results of the Dynamic Characteristics of Methanol- and Ethanol-Water Mixtures at Temperature Range of 35 - 70oc Using an Innovative Technique by Physical Method}

\author{
R.R. Dawam \\ Lecturer, Department of Physics, University of Jos, Nigeria \\ S.B. Fierkwap \\ Assistant Lecturer, Department of Physics, University of Jos, Nigeria
}

\begin{abstract}
:
The analysis of some thermodynamic properties of liquid mixtures was reported. The properties of methanol-water mixture, ethanol-water mixtures such as; density, viscosity, activation energy, enthalpy and entropy were evaluated. These parameters were investigated at different temperatures and constant atmospheric pressure using an innovative technique designed and constructed for the purpose of analysis of water-mixtures in solutions. The activation energy of water, methanol, ethanol, methanol-water mixture and ethanol-water mixture were 14.49 $\pm 0.17,9.05 \pm 0.11$, $14.92 \pm 0.21,12.17 \pm 0.21$ and $14.73 \pm 0.09 \mathrm{~kJ} . \mathrm{mol}^{-1}$ respectively. The corresponding enthalpy and entropy of the liquids were also calculated from the experimentally determined parameters.
\end{abstract}

Keywords: Viscosity, thermodynamics, activation energy, liquid mixtures

\section{Introduction}

The increasing use of fluid in many industrial processes has greatly created the need for extensive research on the thermodynamic transport properties of their mixtures [1].The mixing of different solvents results in the formation of solutions which are different from the ideal [2]. Most of the mixtures are non-ideal and show peculiar behaviours, and the interpretation of non-idealist is an interesting area especially when there is argument with new concepts of friccohesity $[3,4]$. Thermodynamic properties of multi-component liquid mixtures and their analysis is an important subject. Properties such as density, viscosity, temperature, activation energy, enthalpy and entropy are of interest in both theoretical and practical applications. A systematic study of the main excess thermodynamic properties (Gibbs energy, enthalpy) of asymmetric multi-component mixtures of organic molecules, representing different types of molecular interactions was carried out. The transport properties of liquids or liquid mixtures have wide applications in the field of physical sciences, chemical sciences, material sciences and engineering field [5]. Viscosity and density of multi-component mixtures are needed in many chemical engineering calculations involving heat and mass transfer. All these are thermodynamic and transport properties that are important in most design processes. The experimental data of thermodynamic properties of liquid mixture provides useful information about the molecular interaction and can be used to test thermodynamic models [6]. The viscosities of most pure liquids at standard temperature and pressure are well documented, and a number of studies have looked at pressure effects on the viscosity of pure liquids [7]. De and Dikko [8] studied the effect of water concentration on water-ethanol mixtures by using this technique.

Alcohol serves as a simple example of biological and industrially important amphiphilic materials that exist in the liquid state which is due to the hydrogen bonding of their $\mathrm{O}-\mathrm{H}$ group [9]. They are polar and self-associated liquids. The dipolar association of alcohols decreases when they are mixed with aromatic hydrocarbons due to some specific intermolecular interactions between the alcohol and an aromatic hydrocarbon [10, 5] Precise analysis of water content in the mixture of alcohol product are required in the brewery or chemical industry. Some sophisticated technique such as Mass Spectrometry, Spectrophotometry. High Pressure Liquid Chromatography is too expensive to be used in less developed areas for researches. These techniques require experts to operate them and constant power supply is also required to analyse impurity contents of a sample. Therefore the construction of a simple and yet reliable equipment for the analysis of contents of a liquid is of paramount importance [11]. This report is concerned with the viscosity and thermodynamic properties of liquid mixtures using an innovative technique constructed for the analysis of purity of water mixtures. The main aim is to show that the technique can be employed for teaching and research purposes and to determine some thermodynamic properties that cannot be directly measured experimentally. 


\section{Experimental Details}

\subsection{Density}

The densities of the liquids (water, methanol, ethanol, methanol-water mixture, ethanol-water mixture and methanol-ethanol-water mixture) were measured with an oscillation U-tube densitometer at temperatures from $30-70{ }^{\circ} \mathrm{C}$ with Peltier temperature control.

\subsection{Viscosity}

The viscosities of the liquids were investigated using the apparatus described by De and Dikko [8] and modified with temperature controller by Dikko. The operation of the instrument is similar to the falling-body viscometer, in which the viscosity of a fluid is determined by the time it takes a body to fall to a reference position in the fluid of interest related by

$\eta_{2}=\left(\eta_{1} \rho_{2} t_{2}\right) /\left(\rho_{1} t_{1}\right)$

Where $\rho_{2}$ is the density of liquid mixture, $t_{2}$ is the time taken for the liquid to fall to reference position, $t_{1}$ is time of flow of water and $\rho_{1}$ is the density of water. The accuracy in determining $\eta_{2}$ depends strictly on noting the time $t_{1}$ and $t_{2}$. This principle also works perfectly on Oswald viscometer.

A major drawback of this technique is that the density of the fluid of interest at all temperatures must be known exactly, and therefore was determined separately.

\section{Results and Discussion}

\subsection{General Characteristics of Viscosity of Liquid Mixture}

The viscosities of liquid mixtures were investigated as a function of temperature. The temperature ranges from $35-70^{\circ} \mathrm{C}$. Fig 1 (a) shows the graph of viscosity as a function of temperature. It is clearly indicated that as temperature increases viscosity decreases. This trend occurs because of the increased kinetic motion the molecules at higher temperatures which promotes the weakening of intermolecular bonds between adjacent layers.

Density is also affected by temperature. The density decreases with temperature as can be clearly seen in fig 1(b).

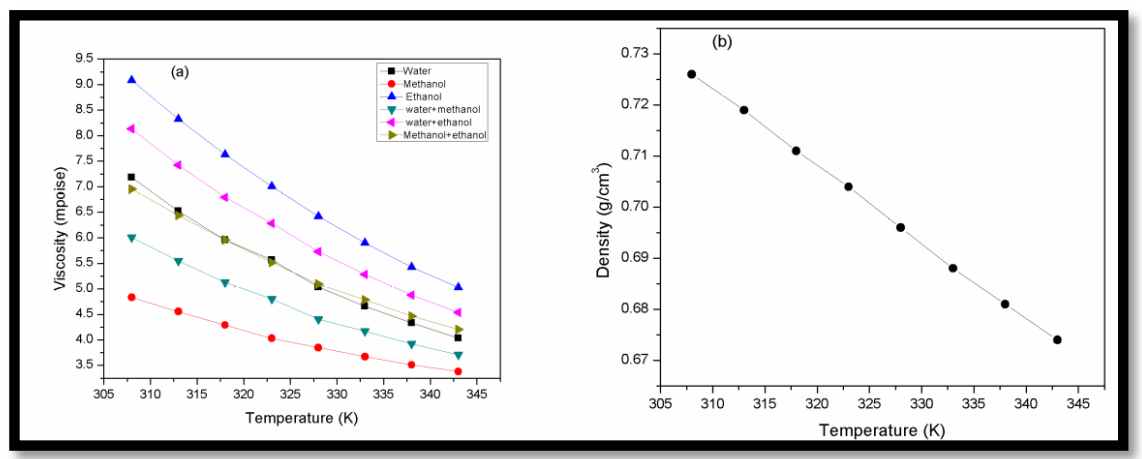

Figure 1: The Plot of Viscosity as a Function of Temperature (A) for All the Samples Under Investigation and (B) Graph of Density against Temperature for Methanol as an Example All Other Liquids Follow the Same Pattern as (B)

\subsection{Viscosity-Temperature Dependence and Activation Energy}

The viscosity of the alcohol-mixture solution was evaluated using the Arrhenius equation given as $\eta=\operatorname{Aexp}(\mathrm{Ea} / \mathrm{RT})$

Where $\eta$ is the viscosity of the solution under the investigation, Ea is the activation energy in (J.mol-1 ${ }^{-1}$, T is the absolute temperature, $\mathrm{A}$ is the pre-exponential factor of the Arrhenius equation for the mixture and $\mathrm{R}$ is the gas constant [12]. Theoretically, the plot of $\ln (\eta)$ against $(1 / \mathrm{RT})$ is linear with slope equal to Ea and the constant A can be evaluated from the intercept on the $\mathrm{y}$-axis as $\mathrm{A}=\exp$ (Intercept). The values of the activation energy of the liquid mixtures are presented in table 1 . These values were compared with the literatures and were found consistent with the results of other researchers, e.g. [4].

Fig. 2 shows the plot of viscosities against temperatures. It shows that ethanol has higher viscosity than pure water, while water in turn has higher viscosity than methanol. It is also seen from Fig.2, that the viscosity of a mixture of ethyl and methyl alcohols and water is controlled mostly by ethyl alcohol and methyl alcohol since the temperature variation of viscosity of the mixture is the same as that of the mixture of ethanol and methanol. This feature is quite interesting and might throw light on the molecular modeling of the mixing of different molecules and formation of barrier heights with activation energy that the molecules have to overcome in flowing motion. 


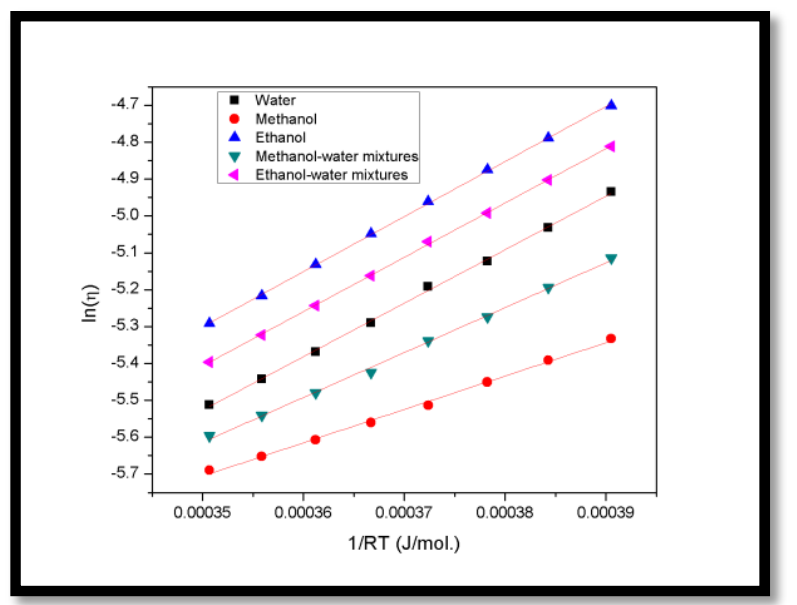

Figure 2: The Graph of Ln (H) Against 1/RT Used for

Evaluation of Activation Energy of

Liquid Mixtures

\subsection{Thermal Expansion Coefficient}

Since the experiments the involve applications of heat by varying the temperature of the liquids from $35-70^{\circ} \mathrm{C}$, the liquid-mixture under investigation may undergo some expansion, according to the definition of the isobaric thermal expansivity which accounts for the variation of temperature with density [12]. It is important to note that all parameters have to be evaluated at the same pressure and thus it is given as;

$\alpha=-\frac{1}{\rho}\left(\frac{d \rho}{d T}\right)_{\mathrm{P}}$

$-\ln \left(\frac{\rho}{\rho o}\right)=\alpha \mathrm{T}+\mathrm{C}$

where $\alpha$ coefficient of thermal expansion can be estimated from the slope of plot of $-\ln \left(\frac{\rho}{\rho o}\right) \quad$ against T. Eq. (4) is the solution of Eq.(3) which is used in the calculation of the thermal expansivity of the liquid and liquid mixtures in this investigation. The plot is expected to be a linear variation. The values of the thermal expansion coefficients for the solutions are summarized in the table 1 for all the samples.

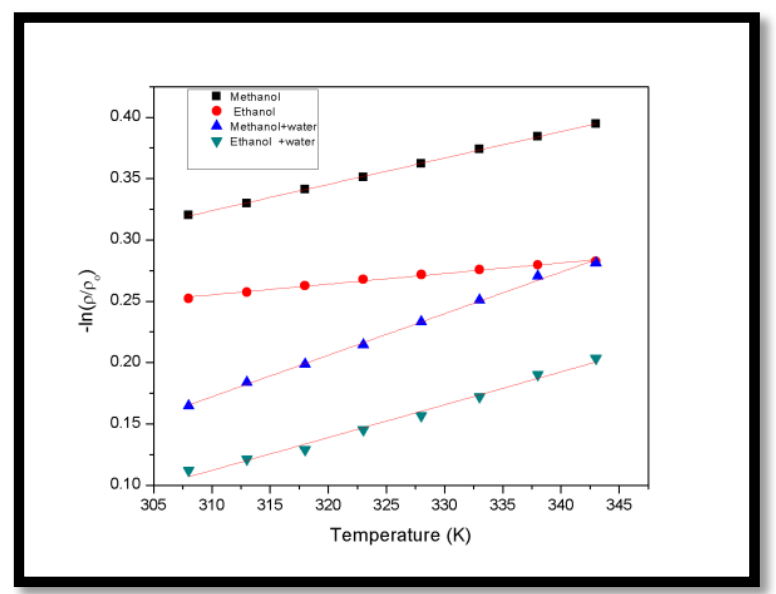

Figure 3: The Graph of - Ln $\left(\frac{\rho}{\rho o}\right)$ Against T Used for Evaluation of

Thermal Expansivity Coefficients of Liquid Mixtures

\subsection{Thermodynamics Properties of Viscous Flow}

Another important relation of temperature dependence of viscosity as proposed by Eyring's theory [13] of viscous flow of liquid can be express as

$\eta=\left(\frac{h N_{A}}{V}\right) \exp \left(\frac{\Delta G}{R T}\right)$

where $\eta$ is the viscosity of the liquid, $\mathrm{V}$ is the molar volume of the mixture, $\mathrm{h}$ is planks' constant, $\mathrm{N}_{\mathrm{A}}$ is Avogadro's number, $\mathrm{R}$ is the gas constant, $\mathrm{T}$ is the absolute temperature and $\Delta \mathrm{G}$ is the molar Gibb's free energy of the activation. When two miscible liquids are brought into contact, they mix and form a homogeneous mixture or solution. This process involves entropy and enthalpy which are related to Gibbs free energy of activation as; 
Putting Eqn (6) into Eqn (5)

$R \ln \left(\frac{\eta V}{h N_{A}}\right)=\left(\frac{\Delta H}{T}\right)-\Delta \mathrm{S}$

Again, the plot of $R \ln \left(\frac{\eta V}{h N_{A}}\right)$ against the reciprocal of temperature is linear with the slope $\Delta \mathrm{H}$ and the intercept equal to $\Delta$ S. Fig 4 , shows the graphs. The corresponding values of enthalpy and entropy are tabulated in table 1.

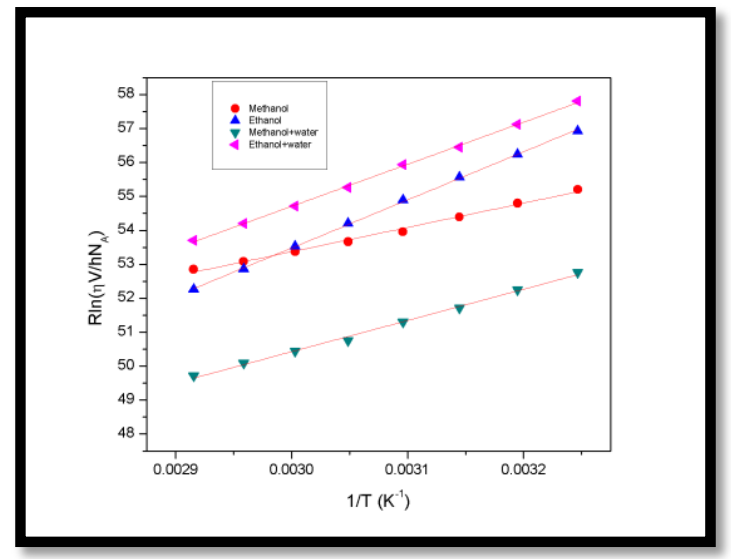

Figure 4: The Graph Of Rln $\left(\frac{\eta V}{h N_{A}}\right)$ Against 1/T Used For Estimation of Change in Enthalpy and Entropy of Liquid Mixtures

\section{Conclusion}

\begin{tabular}{|c|c|c|c|c|}
\hline Samples & Ea (kJ.mol) & $\Delta \mathrm{H}(\mathrm{kJ}: \mathrm{mol})$ & $\Delta S\left(\mathrm{JK}^{-1} \mathrm{~mol}^{-1}\right)$ & $\alpha 10^{-5}\left(K^{-1}\right)$ \\
\hline Water & $\begin{array}{c}14.49 \pm 0.17 \\
15.51[4]\end{array}$ & & & \\
\hline Methanol & $\begin{array}{c}9.05 \pm 0.11 \\
11.02[4]\end{array}$ & $7.16 \pm 0.22$ & $-31.90 \pm 0.68$ & $215.0 \pm 1.70$ \\
\hline Ethanol & $\begin{array}{l}14.92 \pm 0.21 \\
15.8 \pm 0.8[4]\end{array}$ & $14.17 \pm 0.13$ & $-10.97 \pm 0.39$ & $86.8 \pm 3.36$ \\
\hline Methanol+water & $12.17 \pm 0.21$ & $9.20 \pm 0.24$ & $22.82 \pm 0.72$ & $339.0 \pm 6.68$ \\
\hline Ethanol+water & $14.73 \pm 0.09$ & 12.38_0.14 & $-17.75 \pm 0.44$ & $268.0 \pm 11.70$ \\
\hline
\end{tabular}

Table 1: The Experimentally Determined Values of Activation Energy (Ea), Enthalpy $(\mathrm{H})$,

Entropy (S) and Thermal Expansivity (A) Measured for Pure Liquid Samples and Their Mixtures.

The viscosities and densities of the liquid mixture have been reported. The viscosities, densities at temperature range from $35-70^{\circ} \mathrm{C}$ was used to calculate some thermodynamic properties of the liquid mixtures. The results of the thermodynamics were presented in table 1. The emission spectra obtained by Guo et al. [14] reveal that the water and alcohol molecules in solution form complex hydrogen-bonded networks and mix very little at the microscopic level. The results show that the structure of liquid methanol at room temperature is a combination of rings and chains, each made up of either 6 or 8 methanol molecules. When water is added, the methanol chains interact with varying numbers of water molecules. These bridging water molecules bend the chains into open-ring structures that are stable because their gluelike hydrogen bonds are saturated. This means that the mixing of alcohol and water on the microscopic level is incomplete no matter how long you wait. At the molecular level, very little mixing of alcohol and water occurs in solution. Instead, chains of methanol molecules react with clusters of water molecules to form stable open-ring structures, which lower the solution's entropy as can be seen from the structure below.

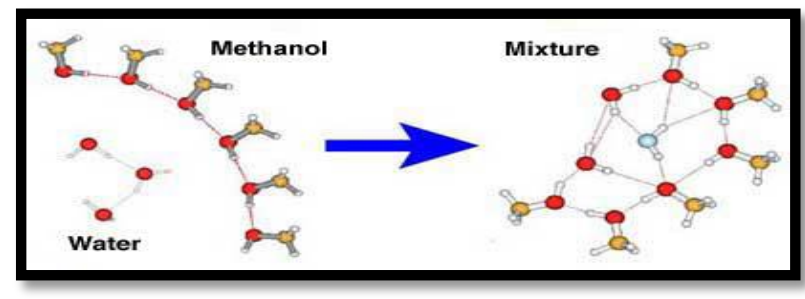

Figure 5 


\section{References}

i. D. M. Jain, V. Shah, S. Rabadiya, S. Oswal, Viscosity and excess molar volume of binary mixtures of methanol with n-butylamine and di-n-butylamine at 303.15, 313.15 and 323.15k. characterization in terms of eras model, Journal of Molecular Liquids 144 (2009) 65 -70.

ii. G. Conti, P. Gianni, L. Lepori, E. Matteoli, Volumetric study of(2-methoxyethanol+tetrahydrofuran+cyclohexane) at $\mathrm{t}=298.15 \mathrm{k}$, The Journal of Chemical Thermodynamics 35 (2003) 503 -518.

iii. M. Bhuiyan, M. Uddin, Excess molar volumes and excess viscosities for mixtures of n,n dimethylformamide with methanol, ethanol and 2- propanol at di_erent temperatures, Journal of Molecular Liquids 138 (2008) 139 - 146.

iv. Z. Trabelsi, M. Dallel, H. Salhi, D. Das, N. Al-Omair, N. Ouerfelli, On the viscosity arrhenius temperature for methanol + n,n-dimethylformamide binary mixtures over the temperature range from 303.15 to $323.15 \mathrm{k}$, Physics and Chemistry of Liquids 53 (2015) 529-552.

v. S. Purohit, S. S. Suthar, M. Vyas, R. C. Beniwal, Studies on transport behaviour of a binary liquid mixture of ethanol and toluene at 298.15k in terms of viscosity models, AIP Conference Proceedings 1953 (2018) 080015.

vi. S. Ranjbar, K. Fakhri, J. B. Ghasemi, Densities and viscosities of (1-propanol + 1,2 dichloroethane), (1-propanol + benzaldehyde), (benzaldehyde + 1,2-dichloroethane), and (1-propanol + 1,2-dichloroethane+ benzaldehyde) mixtures from $t=288.15 \mathrm{k}$ to $313.15 \mathrm{k}$, Journal of Chemical \& Engineering Data 54 (2009) 3284-3290.

vii. W. Thompson, T. J. Kaiser, J. W. Jorgenson, Viscosity measurements of methanol water and acetonitrilewater mixtures at pressures up to 3500bar using a novel capillary time-of-ight viscometer, Journal of Chromatography A 1134 (2006) $201-209$.

viii. D. K. De, A. Dikko, An innovative technique of liquid purity analysis and its application to analysis of water concentration in alcohol-water mixtures and studies on change of activation energies of the mixtures, Applied Physics Research 4 (2012) 98.

ix. E. D. Dikio, S. M. Nelana, D. A. Isabirye, E. E. Ebenso, Density, dynamic viscosity and derived properties of binary mixtures of methanol, ethanol, n-propanol, and n-butanol with pyridine at $\mathrm{t}=(293.15,303.15,313.15$ and 323.15$)$ k, Int. J. Electrochem. Sci 7 (2012) 11101-11122.

x. S. C. Bhatia, R. Rani, R. Bhatia, Viscosities, densities, speeds of sound and refractive indices of binary mixtures of oxylene, m-xylene, p-xylene, ethylbenzene and mesitylene with 1-decanol at 298.15 and $308.15 \mathrm{k}$, Journal of Molecular Liquids 159 (2011) 132 -141.

xi. T. Ono, K. Horikawa, Y. Maeda, M. Ota, Y. Sato, H. Inomata, Dynamic properties of methanolwater mixtures at the temperatures up to $476.2 \mathrm{k}$ and at high pressures via molecular dynamics simulation, Fluid Phase Equilibria 420 (2016) $30-35$.

xii. P. Gmez-lvarez, D. Gonzlez-Salgado, J.-P. Bazile, D. Bessieres, F. Plantier, Excess second-order thermodynamic derivatives of the 2-propanol+water system from $313.15 \mathrm{k}$ to $403.15 \mathrm{k}$ up to $140 \mathrm{mpa}$. experimental and monte carlo simulation study, Fluid Phase Equilibria 35(2013) 7- 26.

xiii. H. Eyring, Viscosity, plasticity, and di_usion as examples of absolute reaction rates, The Journal of chemical physics 4 (1936) 283-291.

xiv. J.-H. Guo, Y. Luo, A. Augustsson, S. Kashtanov, J.-E. Rubensson, D. K. Shuh, H. _Agren, J. Nordgren, Molecular structure of alcohol-water mixtures, Phys. Rev. Lett. 91 (2003) 157401. 